\title{
Chaperone Activity and Dimerization Properties of Hsp90 $\alpha$ and Hsp90 $\beta$ in Glucocorticoid Receptor Activation by the Multiprotein Hsp90/Hsp70-Dependent Chaperone Machinery
}

\author{
Yoshihiro Morishima, Ranjit K. Mehta, Miyako Yoshimura, Miranda Lau, \\ Daniel R. Southworth, Theodore S. Lawrence, William B. Pratt, \\ Mukesh K. Nyati, and Yoichi Osawa \\ Departments of Pharmacology (Y.M., M.Y., M.L., W.B.P., Y.O.) and Radiation Oncology (R.K.M., T.S.L., M.K.N.), University of \\ Michigan Medical School, Ann Arbor, Michigan; and Life Sciences Institute and Departments of Biochemistry and Biophysics, \\ University of Michigan, Ann Arbor, Michigan (D.R.S.)
}

Received March 16, 2018; accepted June 20, 2018

\begin{abstract}
Several hundred proteins cycle into heterocomplexes with a dimer of the chaperone heat shock protein 90 (Hsp90), regulating their activity and turnover. There are two isoforms of Hsp90, $\mathrm{Hsp} 90 \alpha$ and $\mathrm{Hsp} 90 \beta$, and their relative chaperone activities and composition in these client protein $\bullet$ Hsp90 heterocomplexes has not been determined. Here, we examined the activity of human $\mathrm{Hsp} 90 \alpha$ and $\mathrm{Hsp} 90 \beta$ in a purified five-protein chaperone machinery that assembles glucocorticoid receptor (GR)•Hsp90 heterocomplexes to generate high-affinity steroid-binding activity. We found that human $\mathrm{Hsp} 90 \alpha$ and $\mathrm{Hsp} 90 \beta$ have equivalent chaperone activities, and when mixed together in this assay, they formed only GR・Hsp90 $\alpha \alpha$ and $\mathrm{GR} \bullet \mathrm{Hsp} 90 \beta \beta$ homodimers and no $\mathrm{GR} \bullet \mathrm{Hsp} 90 \alpha \beta$ heterodimers. In contrast, GR・Hsp90 heterocomplexes formed in human embryonic kidney (HEK) cells
\end{abstract}

also contain $\mathrm{GR} \bullet \mathrm{Hsp} 90 \alpha \beta$ heterodimers. The formation of $\mathrm{GR} \bullet \mathrm{Hsp} 90 \alpha \beta$ heterodimers in HEK cells probably reflects the longer time permitted for exchange to form Hsp90 $\alpha \beta$ heterodimers in the cell versus in the cell-free assembly conditions. This purified GR-activating chaperone machinery can be used to determine how modifications of Hsp90 affect its chaperone activity. To that effect, we have tested whether the unique phosphorylation of $\mathrm{Hsp} 90 \alpha$ at threonines 5 and 7 that occurs during DNA damage repair affects its chaperone activity. We showed that the phosphomimetic mutant Hsp90 $\alpha$ T5/7D has the same intrinsic chaperone activity as wild-type human $\mathrm{Hsp} 90 \alpha$ in activation of GR steroid-binding activity by the five-protein machinery, supporting the conclusion that T5/7 phosphorylation does not affect $\mathrm{Hsp} 90 \alpha$ chaperone activity.

\section{Introduction}

Heat shock protein 90 (Hsp90) is a highly conserved and essential stress protein that is expressed in all eukaryotic cells, where it regulates the activity and turnover of hundreds of proteins called Hsp90 "clients" (Pratt and Toft, 2003; Taipale et al., 2010). The regulation is both ATP-dependent and dynamic in that proteins are continuously cycling into and out of complexes with Hsp90. The heterocomplexes are formed by a multiprotein chaperone machinery that was discovered during studies of Hsp90 regulation of steroid receptors (Pratt and Toft, 2003; Pratt et al., 2008).

The glucocorticoid receptor (GR) must be in a complex with Hsp90 to have high-affinity steroid-binding activity. Incubation of Hsp90-free GR with concentrated lysates from eukaryotic

This work was supported by National Institutes of Health [Grant GM077430 to Y.O.], [Grant CA131290 to M.K.N.], [Grant CA097248 project IV to M.K.N.], and [Grants GM109896, GM110001A to D.R.S.]. This project is a component of the University of Michigan Medical School's Protein Folding Disease Research Initiative.

https://doi.org/10.1124/mol.118.112516. cells causes the ATP-dependent formation of GR•Hsp90 complexes and restores high-affinity steroid-binding activity. During the 1990s, the components for generating steroid-binding activity were isolated and reconstituted to yield an efficient Hsp90 heterocomplex assembly system of five proteins-Hsp90, Hsp70, Hsp organizer protein (Hop), Hsp40, and p23 (Pratt and Toft, 2003). As cycling with Hsp90 is required for high-affinity glucocorticoid-binding activity in cells, this system affords a physiologically meaningful assay of the intrinsic chaperone activity of Hsp90.

The Agard Lab (University of California, San Francisco) has published a number of cryo-electron microscopy studies showing how the proteins in the Hsp90 chaperone machinery interact and the mechanism of GR・Hsp90 heterocomplex assembly (Southworth and Agard, 2011; Kirschke et al., 2014), but there are details of the active complex that have not yet been worked out. For example, there are two isoforms of human Hsp90, Hsp90 $\alpha$ (gene Hsp90AA1) and Hsp90 $\beta$ (gene Hsp90AB1), that are 86\% homologous in amino acid sequence and are expressed from separate genes, with both isoforms being commonly referred to as Hsp90 (Sreedhar et al., 2004).

ABBREVIATIONS: ATM, ataxia telangiectasia mutated; ATR, ataxia telangiectasia and RAD3-related; GR, Glucocorticoid receptor; HEK, human embryonic kidney; Hop, Hsp organizer protein; Hsp90, heat shock protein 90. 
The functions of $\mathrm{Hsp} 90 \alpha$ and $\mathrm{Hsp} 90 \beta$ are thought to be largely redundant, and they have the same ability to activate the progesterone receptor (Chadli et al., 2008). However, some differences clearly exist. Hsp90 $\beta$ is constitutively expressed in all cells, whereas Hsp90 $\alpha$ is induced during stress and its expression is more tissue-specific (Sreedhar et al., 2004). Mice without $\mathrm{Hsp} 90 \alpha$ are apparently normal, but adult males have arrested spermatogenesis, whereas mouse embryos lacking Hsp90 $\beta$ die at implantation (Voss et al., 2000; Grad et al., 2010).

Dimerization of Hsp90 through the C-terminus is required for in vivo function (Wayne and Bolon, 2007). Although it was shown in 1988 that GR・Hsp90 complexes recovered from cells contain both $\alpha$ and $\beta$ isoforms (Mendel and Ortí, 1988), it is not known whether Hsp90 is present as homo- $(\alpha \alpha, \beta \beta)$ or hetero- $(\alpha \beta)$ dimers. Indeed, as far as we know, the heterocomplex composition has not been directly determined for any Hsp90 client protein.

In this study, we show by the five-protein system that human Hsp90 $\alpha$ and human Hsp90 $\beta$ have the same activity in the activation of GR steroid binding and that steroid binding is activated in direct proportion to the number of GR・Hsp90 heterocomplexes formed. Mixing of $\mathrm{Hsp} 90 \alpha$ and $\mathrm{Hsp} 90 \beta$ under our assay conditions did not yield $\alpha \beta$ heterodimers. Expression of FLAG-Hsp90 $\alpha$ in human embryonic kidney (HEK) cells yielded FLAG-Hsp90 $\alpha \bullet$ FLAG-Hsp90 $\alpha$ homodimers and virtually no dimers of FLAG-Hsp90 $\alpha$ with cellular Hsp90 $\alpha$ or Hsp90 $\beta$. In contrast, GR•Hsp90 complexes isolated from HEK cytosol contained GR•Hsp90 $\alpha \beta$ dimers. Furthermore, to illustrate the utility of this five-protein GR・Hsp90 heterocomplex assembly system, we examined the effect of T5/7 phosphorylation of Hsp90 $\alpha$ on its chaperone activity. This phosphorylation occured only on Hsp90 $\alpha$ and it had been unknown whether this directly affects its chaperone activity. We found that the phosphomimetic mutant Hsp90 $\alpha$ T5/7D has the same activity as wild-type human Hsp90 $\alpha$ in activating GR steroid-binding activity by the purified assembly machinery. This leads us to conclude that this unique modification of Hsp90 $\alpha$, which occurs during DNA double strand-break repair, does not affect its chaperone activity.

\section{Materials and Methods}

Materials. Purified mouse monoclonal IgG2a (clone K41009) used to both immunoadsorb and blot hHsp90 $\alpha$ and purified mouse monoclonal IgM (clone K3701) used to immunoblot hHsp90 $\beta$ were purchased from Enzo Life Sciences (Farmingdale, NY). Purified mouse monoclonal IgG2a (clone H9010) used to immunoadsorb hHsp90 $\beta$ was purchased from Thermo Fisher Scientific (Rockford, IL). The FiGR monoclonal IgG used to immunoadsorb the mouse GR was obtained from Dr. Jack Bodwell (Dartmouth Medical School, Lebanon, NH). The BuGR2 monoclonal IgG used to immunoblot the mouse GR was purchased from Affinity Bioreagents (Golden, CO). Immobilon-FL and PureProteome Nickel Magnetic Beads were purchased from MilliporeSigma (Billerica, MA). Rabbit anti-FLAG IgG was from MilliporeSigma (St. Louis, MO). $\left[1,2,4,6,7-{ }^{3} \mathrm{H}\right]$ Dexamethasone $(77 \mathrm{Ci} / \mathrm{mmol}$ ) was from Perkin Elmer (Waltham, MA). The pSV2Wrec plasmid encoding full-length mouse GR was kindly provided by Dr. Edwin Sanchez (University of Toledo College of Medicine and Life Sciences, Toledo, OH). The FLAG-hHsp90 $\alpha$ vector was received as a gift from Dr. Len Neckers (National Cancer Institute). pET151-dTOPO-Hsp90 $\alpha$ and Hsp90 $\beta$ expression vectors (Southworth Lab, Ann Arbor, MI) were used as a template for site-directed mutagenesis. For the T5/7A hHsp90 $\alpha$ construct, site-directed mutagenesis was performed using sense primer 5'-CTTCACCCCTGAGGAAGCCCAGGCCCAAGACCAACCGATG-3' and antisense primer 5'-CATCGGTTGGTCTTGGGCCTGGGCTTCCTCAGGGGTGAAG- $3^{\prime}$. For the T5/7D hHsp90 $\alpha$ construct, site-directed mutagenesis was performed using sense primer $5^{\prime}$ CTTCACCCCTGAGGAAGACCAGGACCAAGACCAACCGATG-3' and antisense primer 5'-CATCGGTTGGTCTTGGTCCTGGTCTTCCTCAGGGGTGAAG-3'.

Cell Culture. HEK cells were grown in Dulbecco's modified Eagle's medium (DMEM) and 10\% bovine calf serum. HEK cells were harvested into Hanks' buffered saline solution. Cells were centrifuged and pellets were washed in Hanks' buffered saline solution. Cells were then resuspended in HEM buffer (10 mM HEPES, pH 7.4, $1 \mathrm{mM}$ EDTA, and $20 \mathrm{mM}$ sodium molybdate) and Dounce-homogenized, and cytosol was prepared as described previously (Kanelakis and Pratt, 2003). Mouse GR was expressed in Sf9 cells as described (Morishima et al., 2000).

Transient Transfection of Mouse GR. HEK cells were grown as monolayers (162-cm ${ }^{2}$ culture flasks to $\sim 50 \%$ confluency), washed, and treated with $25 \mu \mathrm{g}$ of plasmid DNA and $75 \mu \mathrm{l}$ of TransFast transfection reagent (Promega, Madison, WI). After 1 hour, $10 \mathrm{ml}$ of DMEM with $10 \%$ bovine calf serum was added, and the transfection was continued for 48 hours.

Immunoadsorption of GR and GR•Hsp90 Heterocomplex Assembly. Receptors were immunoadsorbed from aliquots of $50 \mu \mathrm{l}$ (for measuring steroid binding) or $100 \mu \mathrm{l}$ (for Western blotting) of Sf9 cell cytosol as described (Morishima et al., 2000). For heterocomplex reconstitution with purified proteins, immunopellets containing GR stripped of chaperones were incubated with $20 \mu \mathrm{g}$ of purified Hsp90, $15 \mu \mathrm{g}$ of purified rabbit Hsp70, $0.6 \mu \mathrm{g}$ of purified human Hop, $6 \mu \mathrm{g}$ of purified human p23, $0.125 \mu \mathrm{g}$ of purified yeast YDJ-1 adjusted to $55 \mu \mathrm{l}$ with HKD buffer (10 mM Hepes, $\mathrm{pH} 7.4$, $100 \mathrm{mM} \mathrm{KCl}, 0.1 \mathrm{mM}$ dithiothreitol) containing $20 \mathrm{mM}$ sodium molybdate and $5 \mu \mathrm{l}$ of an ATP-regenerating system (50 mM ATP, $250 \mathrm{mM}$ creatine phosphate, $20 \mathrm{mM}$ magnesium acetate, and $100 \mathrm{IU} / \mathrm{ml}$ creatine phosphokinase). The assay mixtures were incubated for 20 minutes at $30^{\circ} \mathrm{C}$ with shaking and subsequently assayed for GR-associated Hsp90 and steroid-binding capacity as described (Morishima et al., 2000).

Gel Electrophoresis and Western Blotting. Immune pellets were run on $12 \%$ SDS-polyacrylamide gels and transferred to Immobilon-FL membranes. The membranes were probed with $1.0 \mu \mathrm{g} / \mathrm{ml} \mathrm{BuGR2} \mathrm{for} \mathrm{GR,} 0.4 \mu \mathrm{g} / \mathrm{ml}$ anti-Hsp90 $\alpha$ IgG2a for hHsp90 $\alpha$, and $1.0 \mu \mathrm{g} / \mathrm{ml}$ anti-Hsp90 $\beta$ IgM for hHsp90 $\beta$ for 1 hour at room temperature. The immunoblots were then incubated a second time with IRDye 800CW-conjugated goat anti-mouse IgG $(\mathrm{H}+\mathrm{L})$, IRDye $800 \mathrm{CW}$-conjugated goat anti-mouse IgG2a ( $\gamma 2 \mathrm{a}$ chain specific), and IRDye 680LT-conjugated goat anti-mouse IgM ( $\mu$-chain specific) for GR, hHsp90 $\alpha$, and $\mathrm{hHsp} 90 \beta$, respectively, for 1 hour at room temperature. Washed membranes were scanned by Odyssey Fc Imaging System (LI-COR Biosciences, Lincoln, NE) and the immunoreactivity was quantitated by Image Studio software within a linear dynamic range for each protein.

Protein Purification. Hsp70, Hop, YDJ-1 (the yeast homolog of Hsp40), and p23 were purified as described by Kanelakis and Pratt (2003). DNAs encoding wild-type hHsp90 $\alpha$, T5/7A mutant hHsp90 $\alpha$, T5/7D mutant hHsp90 $\alpha$, and Hsp90 $\beta$ were cloned into the pET151 vector with a His ${ }_{6}$-tag and tobacco etch virus cleavage site (Invitrogen/Thermo Fisher Scientific). Protein was purified as described by Southworth and Agard (2008) by nickel affinity (Ni-NTA Superflow; QIAGEN, Hilden, Germany), ion exchange (HiTrap Q FF; GE Healthcare, Chicago, IL), and size exclusion (Superdex 200 pg; GE Healthcare) chromatographies. Tobacco etch virus cleavage of the $\mathrm{His}_{6}$-tag was performed prior to the size exclusion column step. The mixture of $\mathrm{rHsp} 90 \alpha$ and $\mathrm{rHsp} 90 \beta$ was purified from rabbit reticulocyte lysate as described by Kanelakis and Pratt (2003). 


\section{Results}

Equivalence of Human Hsp90 $\alpha$ and Hsp90ß in GR•Hsp90 Heterocomplex Assembly. To bind steroid with high affinity, the GR must be assembled into a heterocomplex with Hsp90 (Pratt and Toft, 2003). Figure 1a shows that incubation of an Hsp90-free immunopellet with a mixture of Hsp70, Hop, Hsp40, and p23 did not yield steroid-binding activity, whereas addition of purified human Hsp90 $\alpha$ or human Hsp90 $\beta$ to the mix did yield steroid-binding activity. Human Hsp90 $\alpha$ and Hsp90 $\beta$ yield equivalent GR steroidbinding activity that is similar to the mixture of Hsp90 $\alpha$ and Hsp90 $\beta$ that we purify from rabbit reticulocyte lysate. Figure $1 \mathrm{~b}$ shows the formation of GR・Hsp90 heterocomplexes with three amounts of human Hsp90 $\alpha$ and human Hsp90 $\beta$. To obtain the data of Fig. 1, c and d, GR・Hsp90 heterocomplexes were prepared as in Fig. $1 \mathrm{~b}$ and split, with one half assayed by immunoblotting and scanned for the relative amount of Hsp90 bound to the GR (Fig. 1c) and the other half assayed for steroid-binding (Fig. 1d). Clearly, the relative amount of steroid bound reflects the relative amount of $\mathrm{GR} \cdot \mathrm{Hsp} 90$ heterocomplex formed, and $\mathrm{hHsp} 90 \alpha$ and hHsp90 $\beta$ had equivalent chaperone activities in this assay.

Formation of Homodimers versus Heterodimer of Human Hsp90 $\alpha$ and Hsp90 $\beta$. We determined that addition of a 1:1 mixture of hHsp90 $\alpha$ and hHsp90 $\beta$ had the same ability to generate GR steroid-binding activity by the five-protein

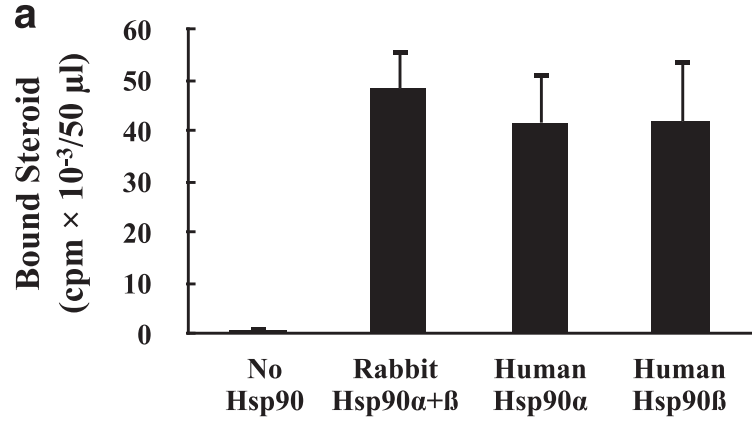

b
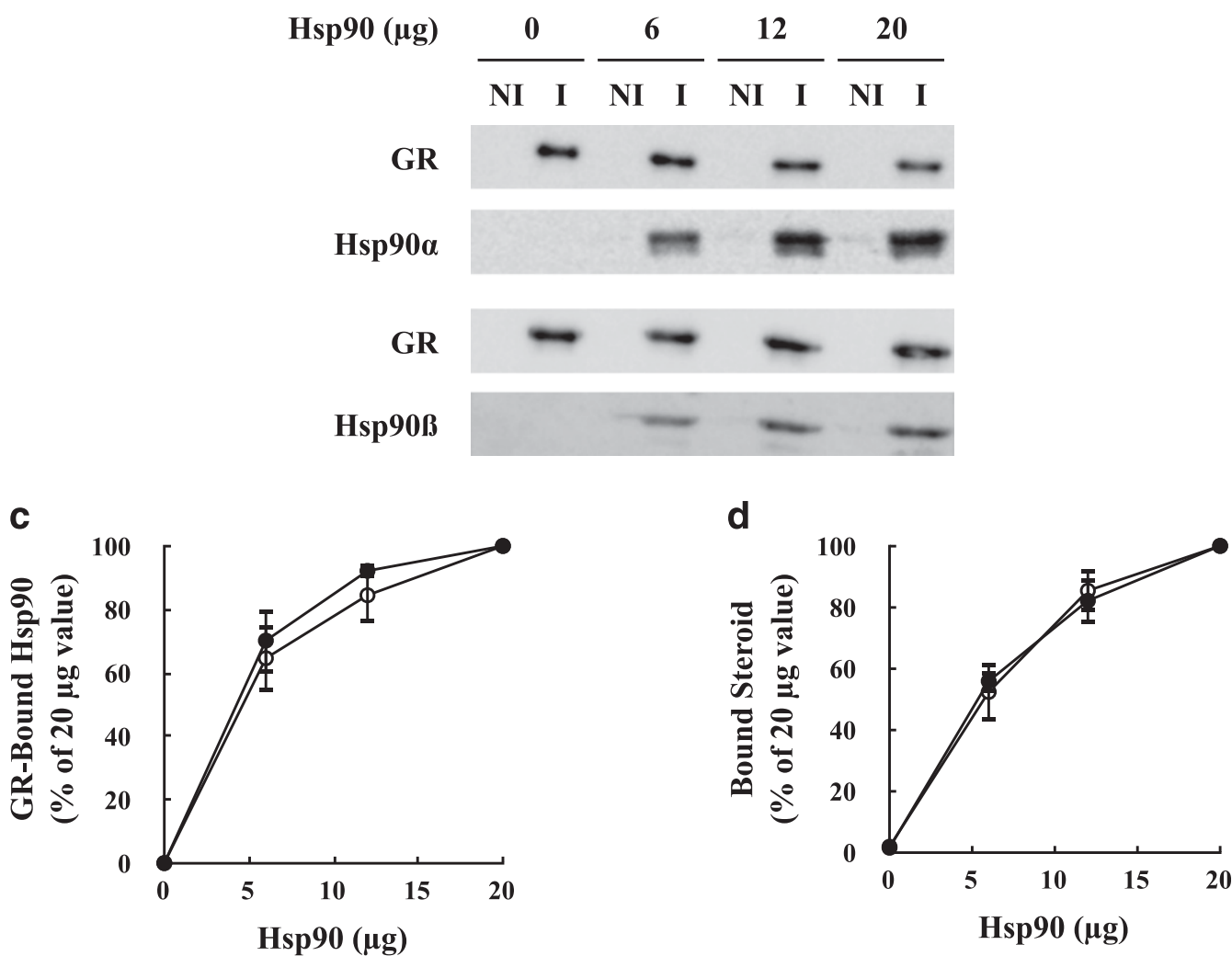

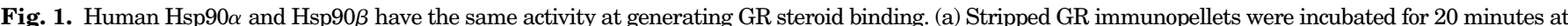

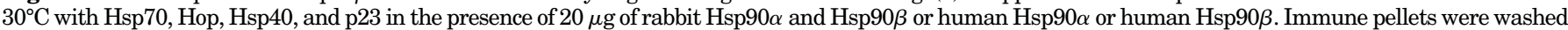

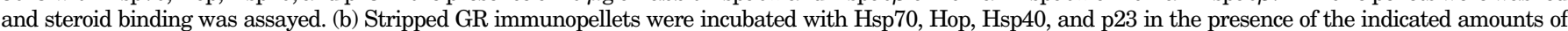

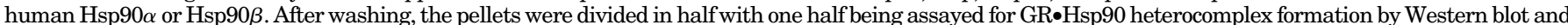



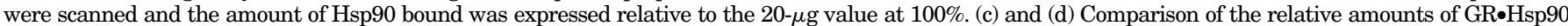
heterocomplex and steroid binding, respectively. (O) Hsp90 $\alpha$; ( $)$ Hsp90 $\beta$. The values for (a), (c), and (d) represent the mean \pm S.D. for three experiments. 
mix as the same amount of hHsp90 $\alpha$ or hHsp90 $\beta$ alone (data not shown). We wanted then to know whether under our GR•Hsp90 heterocomplex assembly conditions any Hsp90 $\alpha \beta$ heterodimers were formed. A great species difference in stability of Hsp90 dimers has been demonstrated. In the absence of ATP, yeast Hsp90 at low concentrations dissociated and could be found in a monomer-dimer equilibrium (Richter et al., 2008). In contrast to the rapid subunit exchange of yeast Hsp90, a lack of heterodimerization between hHsp90 $\alpha$ and hHsp90 $\beta$ was reported by Minami et al. (1994), apparently because of a lack of dissociation of hHsp90 $\alpha$ homodimers (Sreedhar et al., 2004). Under conditions of increased ionic strength and temperature, Richter et al. (2001) were able to achieve some subunit exchange to form hHsp90 $\alpha \bullet$ hHsp90 $\beta$ heterodimers. In the experiment of Fig. 2, His-tagged hHsp90 $\beta$, which was as active as wild-type hHsp90 $\beta$ at GR activation by the five-protein system (data not shown), and an equal amount of purified $\mathrm{hHsp} 90 \alpha$ were mixed under the buffer conditions we used for GR•Hsp90 heterocomplex assembly. The mixture was incubated at $30^{\circ} \mathrm{C}$, and at various times, aliquots were adsorbed to a nickel matrix with all of the His-tagged Hsp90 $\beta$ recovered in the pellet. No Hsp90 $\alpha$ was coprecipitated after 20 minutes ( 0.3 hours). We did detect a small amount $(\sim 9 \%)$ of $\mathrm{hHsp90} \alpha \bullet \mathrm{His}-\mathrm{Hsp} 90 \beta$ heterodimer formation when the incubation was extended to 8 hours. A control sample containing purified hHsp90 $\alpha$ and hHsp90 $\beta$ with no His-tagged hHsp90 $\beta$ was treated similarly to show the background level of hHsp90 $\alpha$ in the pellet, which is at the level of the 0 time value for the His-tagged hHsp90 $\beta$ nickel adsorption. We concluded that heterodimers of hHsp90 do not form during our usual assay conditions (20 minutes at $30^{\circ} \mathrm{C}$ ) but form very slowly during incubation of several hours. Thus, when we incubate with a 1:1 mix of hHsp90 $\alpha$ and hHsp90 $\beta$ to assemble GR॰hHsp90 heterocomplexes, we were forming only GR•hHsp90 $\alpha \alpha$ and GR•hHsp90 $\beta \beta$ and no GR॰hHsp90 $\alpha \beta$ complexes.

Expression of FLAG-hHsp90 $\alpha$ in HEK Cells Suggests Homeostatic Regulation of hHsp90 and Only Trace $\alpha \beta$ Heterodimer Formation. Although hHsp90 $\alpha \beta$ heterodimers were not readily formed on mixing of purified proteins under our assay conditions $\left(20\right.$ minutes, $\left.30^{\circ} \mathrm{C}\right)$, it is possible that heterodimers can be formed in cells. To address this possibility, FLAG-tagged hHsp90 $\alpha$ was expressed in HEK cells. As shown in Fig. 3a, as more FLAG-tagged hHsp90 $\alpha$ was
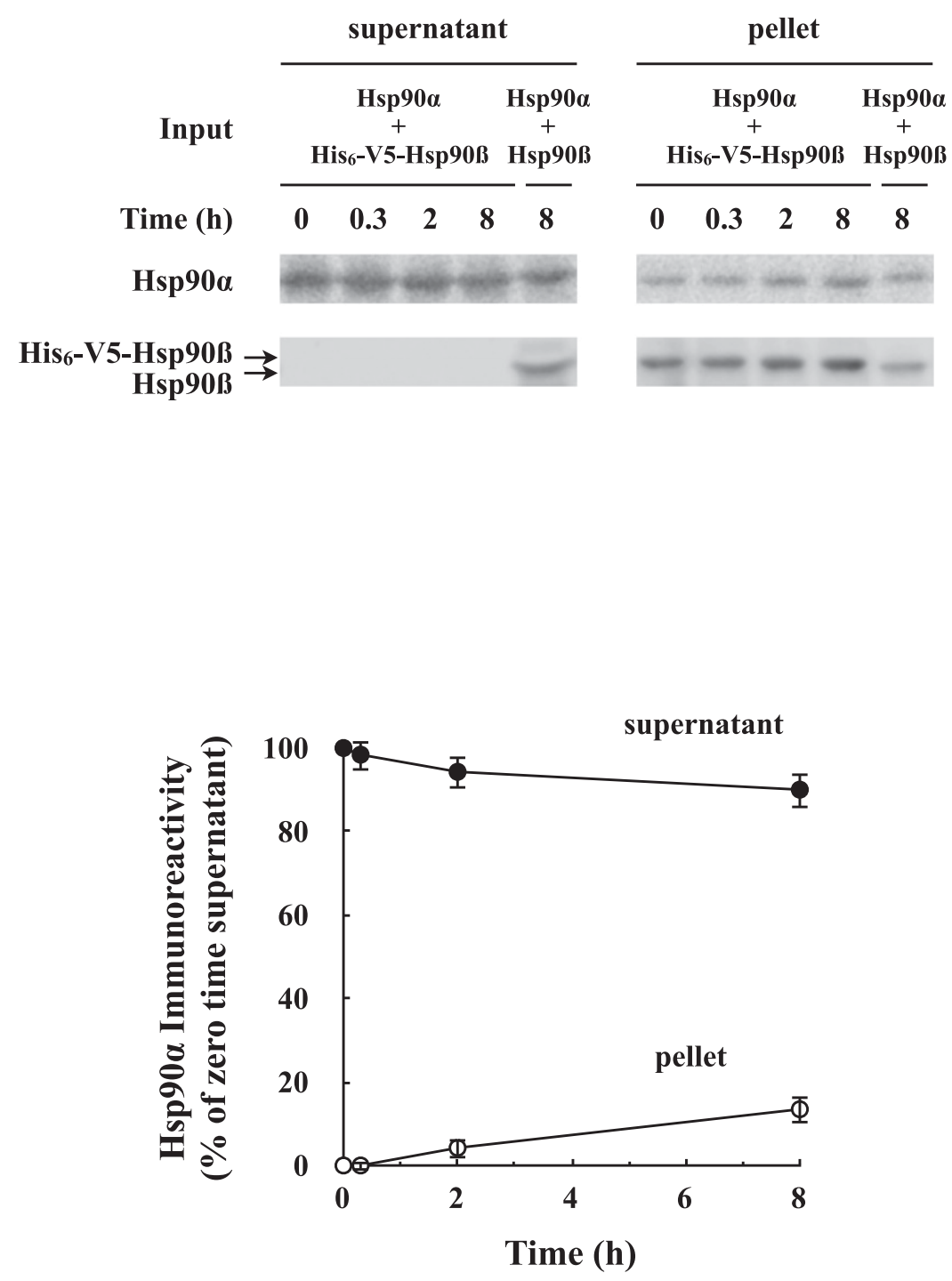

Fig. 2. Mixture of human Hsp90 $\alpha$ with $\mathrm{Hsp} 90 \beta$ yields little heterodimer formation. Equal amounts of purified human $\mathrm{Hsp90} \alpha$ and His-tagged human $\mathrm{Hsp} 90 \beta$ ( $\mathrm{His}_{6}$-V5-Hsp90 $\beta$ ) were incubated for the indicated times at $30^{\circ} \mathrm{C}$ under the same conditions used for GR•Hsp90 heterocomplex assembly. $\mathrm{His}_{6}$-V5-Hsp90 $\beta$ was adsorbed to a nickel matrix and Hsp90 $\alpha$ and Hsp90 $\beta$ were immunoblotted and scanned. The amount of Hsp90 $\alpha$ in a nickel pellet prepared from a control sample with hHsp90 $\alpha$ and hHsp90 $\beta$ without His-tagged Hsp90 $\beta$ was the same as the 0 time pellet prepared from the mixture. The graph presents the means \pm S.D. for three experiments. 
expressed, less endogenous hHsp90 $\alpha$ and hHsp90 $\beta$ was present in the lysate. The decrease in endogenous hHsp90 when FLAG-hHsp90 $\alpha$ was expressed suggests that the total amount of hHsp90 produced in the HEK cell was under homeostatic regulation. It is known from both cell-free (Zou et al., 1998) and cellular (Bharadwaj et al., 1999) studies that Hsp90 is a repressor of the heat shock transcription factor HSF1, but if that accounted for the phenomenon we observed here, we are surprised to see such a decrease in the constitutive isoform hHsp90 $\beta$.

In Fig. 3b, lysate from FLAG-hHsp90 $\alpha$-expressing cells was immunoadsorbed with anti-FLAG and the immunopellet was blotted for both hHsp90 $\alpha$ and hHsp90 $\beta$. Only a small amount of hHsp90 $\beta$ was coimmunoprecipitated, suggesting relatively little $\alpha \beta$ heterodimer formation, but surprisingly almost no endogenous HEK hHsp90 $\alpha$ was in the pellet either. Thus, it appears that the FLAG-hHsp90 $\alpha$ was dimerized almost totally with itself. Sullivan and Toft (1993) noted that newly synthesized in vitro translates of Hsp90 did not complex with endogenous Hsp90, and they suggested that Hsp90 monomers from a single polysome are separated by a short distance relative to monomers on separate polysomes and are thus more prone to interact to form homodimers. This may be the case in HEK cells, where we obtained an abundance of FLAG-hHsp90 $\alpha$ FLAG-hHsp90 $\alpha$ dimers and only trace FLAG-hHsp90 $\alpha \bullet$ endogenous hHsp90 $\alpha$ dimers. We should note that FLAG can dimerize with itself, and this could have contributed to the dimerization of FLAG-Hsp90 $\alpha$ with itself.

Mouse GR Expressed in HEK Cells Is Associated with hHsp90 $\boldsymbol{\alpha} \boldsymbol{\beta}$ Heterodimers. It seems as though some hHsp90 $\alpha \beta$ heterodimers are formed in HEK cells, and we wanted to determine if GR•Hsp90 heterocomplexes formed in HEK cells contain hHsp90 $\alpha \beta$ heterodimers. To approach this question we used two immunoprecipitating antibodies specific for Hsp90 $\alpha$ and Hsp90 $\beta$, respectively. The specificity of immunoprecipitation is shown in Fig. 4a: Purified hHsp90 $\alpha$ or purified hHsp90 $\beta$ was immunoprecipitated with nonimmune, anti-Hsp90 $\alpha$ and anti-Hsp90 $\beta$ antibodies. Clearly, the immunoprecipitation was specific for the antibody targets. As shown in Fig. 4b, purified hHsp90 $\alpha$ and hHsp90 $\beta$

\section{a}

\section{$\begin{array}{llllll}\text { FLAG-Hsp90 } \alpha \text { cDNA }(\mu g) & 0 & 0.25 & 0.5 & 1\end{array}$}

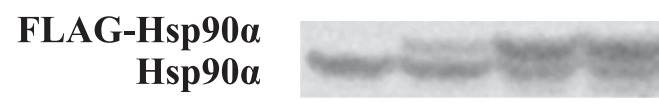

\section{Hsp903}

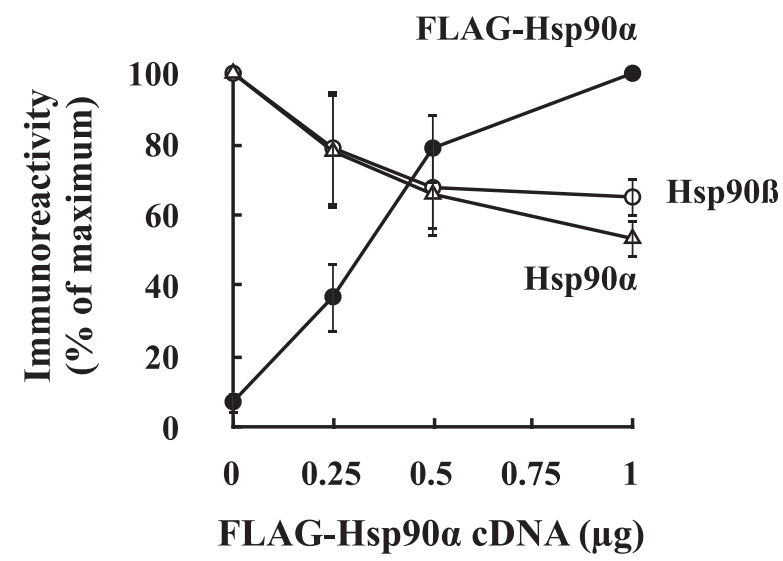

Immunoadsorption

FLAG-Hsp90a
Fig. 3. Expression of FLAG-Hsp90 $\alpha$ in HEK cells suggests that the total amount of Hsp90 is under homeostatic regulation. (a) HEK cells were transfected with FLAG-Hsp90 $\alpha$ cDNA, and 48 hours later, lysates were prepared and immunoblotted for Hsp90 $\alpha$ and Hsp90 $\beta$. The graph shows the mean \pm S.D. of scans for three experiments expressed as the percentage of maximum immunoreactivity. (O) FLAGHsp90 $\alpha,(\triangle)$ endogenous HEK Hsp90 $\alpha,(\bigcirc)$ HEK Hsp90 $\beta$. (b) FLAG-hHsp90 $\alpha$ forms primarily homodimers with itself and not mixed dimers with endogenous HEK Hsp90 $\alpha$ or Hsp90 $\beta$. Lysate prepared as above was immunoadsorbed with anti-FLAG $(I)$ or nonimmune $(N I)$ antibody and immunoblotted with anti-Hsp90 $\alpha$ or anti-Hsp90 $\beta$.
Immunoblotting

anti-Hsp90 $\alpha$

anti-Hsp90ß

\section{FLAG-Hsp90a Hsp90a}

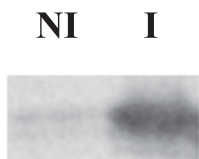

Hsp90ß

\section{input}
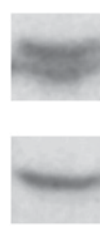

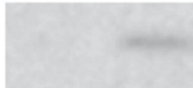


a

$\frac{\text { IP with }}{\text { NI } \underset{\text { Hsp90 }}{\text { anti- }} \underset{\text { Hsp90ß }}{\text { anti- }}}$

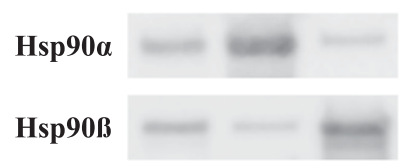

C

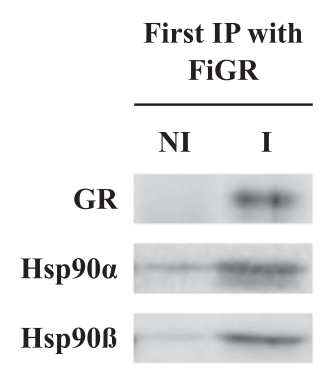

b

NI $\underset{\text { Hsp90a Hsp90ß }}{\text { anti- }}$

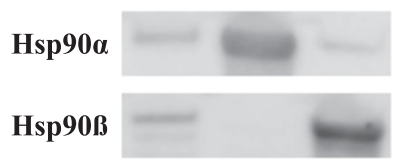

peptide released complex

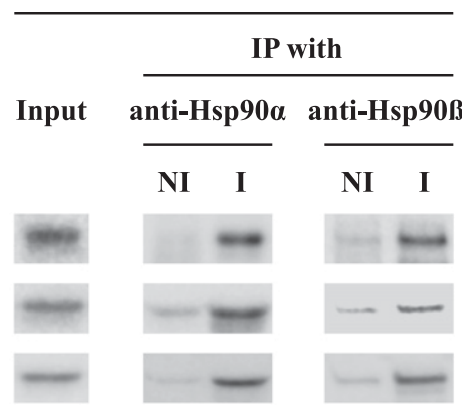

Fig. 4. GR expressed in HEK cells is associated with some Hsp90 $\alpha \beta$ heterodimers. (a) Purified hHsp90 $\alpha$ or hHsp90 $\beta$ were immunoadsorbed with nonimmune $(N I)$, anti-Hsp90 $\alpha$ or anti-Hsp90 $\beta$ antibodies to demonstrate the specificity of immunoprecipitation. (b) Equal amounts of purified hHsp90 $\alpha$ and hHsp90 $\beta$ were mixed and incubated for 20 minutes at $30^{\circ} \mathrm{C}$ in the GR・Hsp90 heterocomplex assembly assay buffer with the ATP regenerating system. The mixture was then immunoprecipitated with nonimmune $(N I)$, anti-Hsp90 $\alpha$, or anti-Hsp90 $\beta$ antibodies, and the immune pellets were blotted for $\mathrm{Hsp} 90 \alpha$ or $\mathrm{Hsp} 90 \beta$. (c) GR॰hHsp90 heterocomplexes formed in HEK cells. HEK cells were transfected with mouse GR cDNA, and 48 hours later, cell lysates were immunoadsorbed with FiGR anti-GR monoclonal $(I)$ or nonimmune $(N I)$ antibody. The washed pellets are First IP. Complexes were released by competition with a peptide encompassing the FiGR epitope (Input) and immunoadsorbed with anti-Hsp90 $\alpha$, anti-Hsp90 3 , or nonimmune antibody. The gels were Western blotted for GR, Hsp90 $\alpha$, and Hsp90 $\beta$. were mixed and incubated 20 minutes at $30^{\circ} \mathrm{C}$ in our GR reconstitution assay buffer. The data confirmed our previous conclusion that no hHsp90 $\alpha \beta$ heterodimers were formed under our GR•Hsp90 heterocomplex assembly conditions. For Fig. 4c, we expressed the mouse GR in human embryonic kidney cells and immunoadsorbed the GR•human Hsp90 heterocomplexes (First IP). The complexes were then released from the antibody beads by competition with a FiGR epitope peptide (Input), as we have previously reported (Murphy et al., 2003). The released complexes were then immunoprecipitated a second time with anti-Hsp90 $\alpha$ or anti-Hsp90 $\beta$, and all samples were immunoblotted for GR, Hsp90 $\alpha$, and Hsp90 $\beta$. As expected from the work of Mendel and Ortí (1988), both Hsp90 $\alpha$ and Hsp90 $\beta$ were present in the first immunoprecipitation with anti-GR antibody. Importantly, after release, the anti-Hsp90 $\alpha$ and the Hsp90 $\beta$ immunoprecipitates contained the GR and both $\mathrm{Hsp} 90 \alpha$ and Hsp90 $\beta$. We conclude that HEK cells form GR・Hsp90 $\alpha \beta$ heterodimers, suggesting some exchange of human Hsp90 $\alpha$ with human Hsp90 $\beta$ in the cell.

Phosphomimetic hHsp90 $\alpha$ T5/7D Has the Same Chaperone Activity as Wild-Type hHsp90 $\alpha$. The purified GR-activating machinery we used here can also be used to determine how modification of an Hsp90 isoform affects chaperone activity. Several laboratories have focused on a unique phosphorylation of $\mathrm{Hsp} 90 \alpha$ at threonines 5 and 7 that occurs during DNA double-strand break repair (Lees-Miller and Anderson, 1989; Quanz et al., 2012; Solier et al., 2012; Elaimy et al., 2016). This Hsp90 $\alpha$ T5/7 phosphorylation is carried out by the members of the phosphatidylinositol 3-kinase-related kinase family [ataxia telangiectasia mutated (ATM), ataxia telangiectasia and RAD3-related (ATR), and DNA-dependent protein kinase (DNA-PK)], which serve as damage sensors that initiated a signaling cascade giving rise to DNA repair, cell cycle arrest, or cell death (Matsuoka et al., 2007; Ciccia and Elledge, 2010). Although it is clear that phosphorylation per se is important for DNA repair, it has not been determined whether phosphorylation of Hsp90 $\alpha$ alters its chaperone activity.

To determine if threonine phosphorylation affects the chaperone activity of hHsp90 $\alpha$, we prepared two varieties of hHsp90 $\alpha$ in which threonines 5 and 7 were converted to alanine or to aspartate, with the latter serving as a phosphomimetic hHsp90 $\alpha$. As shown in Fig. 5 , both mutants of hHsp90 $\alpha$ had the same ability to activate GR steroidbinding activity by the five-protein machinery as wild-type hHsp90 $\alpha$. The equivalence of wild-type and phosphomimetic

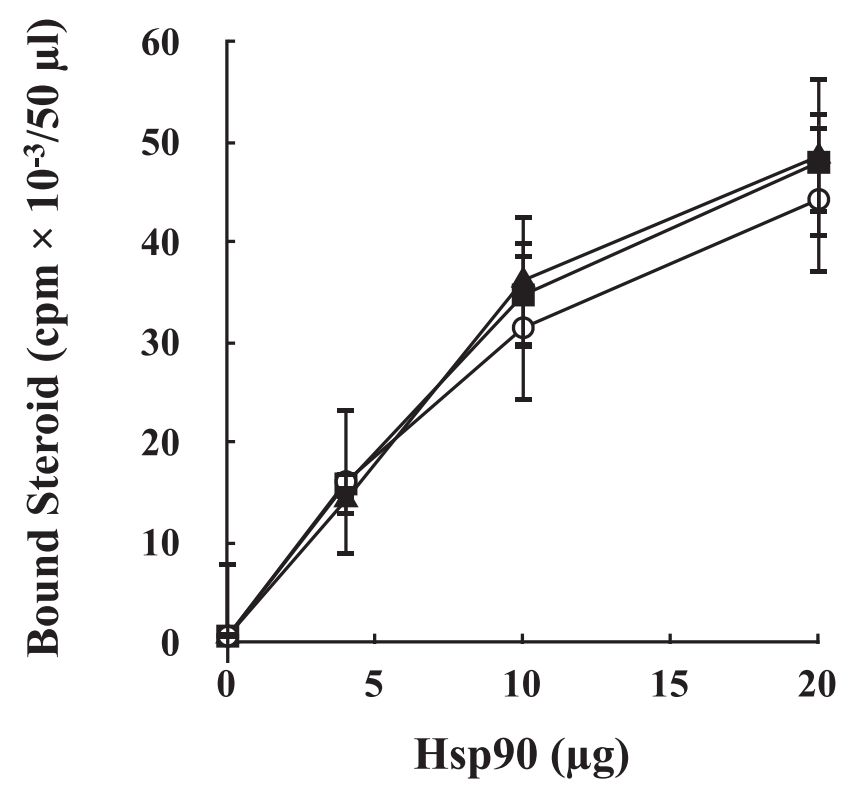

Fig. 5. Phosphomimetic T5/7D-Hsp90 $\alpha$ has the same chaperone activity on the GR as wild-type Hsp90 $\alpha$. Stripped GR immunopellets were incubated for 20 minutes at $30^{\circ} \mathrm{C}$ with Hsp70, Hop, Hsp40, and p23 in the presence of the indicated amounts of human Hsp90 $\alpha(O)$, human Hsp90 $\alpha$ T5/7A (-), or human Hsp90 $\alpha$ T5/7D ( $\mathbf{\Delta})$, and steroid binding was assayed. Data are mean \pm S.D. from three experiments. 
hHsp90 $\alpha$ in this assay suggests that T5/7 phosphorylation does not alter the intrinsic chaperone activity of hHsp90 $\alpha$.

\section{Discussion}

Hsp90 $\beta$ is expressed constitutively at a higher level than Hsp90 $\alpha$ in most tissues, but Hsp90 $\alpha$ is stress-inducible and present at higher levels during stress. This induction of Hsp90 $\alpha$ will increase the $\alpha / \beta$ isoform ratio, and the change will be marked if there are differences in intrinsic chaperone activity between the two isoforms. When expressed as the only Hsp90 of yeast, the $\alpha$ and $\beta$ isoforms of human Hsp90 differ with regard to their capacities for activation in a manner that is very client-protein-dependent (Millson et al., 2007). Because cycling with Hsp90 is required for high-affinity glucocorticoid-binding activity in cells, the five-protein system that we used here provides both a precise and physiologically meaningful assay of the intrinsic chaperone activity of the Hsp90 $\alpha$ and $\beta$ isoforms.

It is quite clear that homodimers of hHsp90 $\alpha$ have the same chaperone activity as homodimers of $h H s p 90 \beta$ as measured by their ability to form GR・Hsp90 heterocomplexes and to activate GR steroid-binding activity when they are present in the five-protein multichaperone machinery (Fig. 1). This strongly suggests an equivalence in their intrinsic physiologic chaperone activities. Although mixture of hHsp90 $\alpha$ and hHsp90 $\beta$ does not readily allow exchange to yield $\alpha \beta$ heterodimer formation, heterodimers can be forced by changing the incubation conditions (Richter et al., 2001) or by greatly increasing the incubation time under the conditions of our GR•Hsp90 heterocomplex assembly (Fig. 2). Thus, when we assembled GR・hHsp90 heterocomplexes with the fiveprotein machinery with both isoforms of human Hsp90 present, we were assembling predominantly GR॰hHsp90 $\alpha \alpha$ and GR॰hHsp90 $\beta \beta$ complexes, with trace amounts, if any, of GR॰hHsp90 $\alpha \beta$ complexes being formed.

The preference for homodimer formation also exists when FLAG-hHsp90 $\alpha$ is expressed in HEK cells, where we find only very little dimer formation with either endogenous hHsp90 $\alpha$ or hHsp90 $\beta$. To us, the most reasonable explanation for this is that of Sullivan and Toft (1993) who suggested that Hsp90 monomers from a single polysome are separated by a short distance relative to monomers on separate polysomes and it is thus more probable that they will interact to form homodimers. The strong binding affinity between hHsp90 $\alpha \alpha$ and hHsp90 $\beta \beta$ homodimer units makes subsequent exchange of subunits to form $\mathrm{hHsp} 90 \alpha \bullet \mathrm{hHsp} 90 \beta$ heterodimers negligible when the purified proteins are mixed in vitro, but in the cell some heterodimers exist, and some GR॰hHsp90 heterocomplexes formed in the cell are GR・hHsp90 $\alpha \beta$ complexes (Fig. 4c). There are probably also GR॰hHsp90 $\alpha \alpha$ and GR・hHsp90 $\beta \beta$ complexes formed in the cell as well. The difference between hHsp90 $\alpha \beta$ heterodimer formation in the cell and lack of heterodimer formation under our purified protein reconstitution assay conditions probably simply reflects the many hours at $37^{\circ} \mathrm{C}$ permitted for exchange in the cell. However, it is possible that post-translational modifications play a role in enabling the formation of hHsp90 $\alpha \beta$ heterodimers in the cell.

Hsp90 is one of the most abundant proteins in unstressed cells where it comprises $1-2 \%$ of cytosolic proteins. We have previously reported that overexpression of Hsp90 in HEK cells increases the level of Hsp90 only 2- to 3 -fold, but that is sufficient to yield a profound stabilizing effect on client protein (neuronal nitric-oxide synthase) turnover (Peng et al., 2012). As we increased expression of FLAG-hHsp90 $\alpha$, we noted a decrease in the levels of endogenous hHsp90 $\alpha$ and hHsp90 $\beta$, suggesting that the total amount of Hsp90 is under homeostatic regulation (Fig. 3a). Although Hsp90 is an established repressor of HSF1 (Zou et al., 1998; Bharadwaj et al., 1999), the homeostatic regulation of Hsp90 we observed here involves both the stress-inducible hHsp90 $\alpha$ and the constitutive Hsp90 $\beta$, suggesting a different form of regulation. Hsp90 and Hsp70 have opposing effects on client protein turnover in HEK cells-Hsp90 inhibits substrate ubiquitination and degradation, whereas Hsp70 promotes ubiquitination and degradation (Peng et al., 2012). Thus, it may be important for general proteostasis that the cell maintain an appropriate ratio of these two naturally abundant components of the chaperone machinery, with homeostatic regulation of total Hsp90 expression being one component of that regulation.

The glucocorticoid receptor is possibly the most studied protein as an Hsp90 client, and to our knowledge, this study of the isoform composition of the GR・Hsp90 complex is unique among the hundreds of established Hsp90 client proteins. From our results, we would predict that GR・Hsp90 heterocomplexes produced in cells contain $\mathrm{Hsp} 90 \alpha \beta$ heterodimers as well as $\mathrm{Hsp} 90 \alpha \alpha$ and $\mathrm{Hsp} 90 \beta \beta$ homodimers, although the exact ratio of the three forms is not known.

Because Hsp90 $\alpha$ T5/7 phosphorylation occurs during DNA double-strand break repair, it has been assumed that the phosphorylation of Hsp90 $\alpha$ must be in some way meaningful. However, the equivalence of wild-type hHsp90 $\alpha$ and the phosphomimetic mutant T5/7D hHsp90 $\alpha$ in their ability to activate GR steroid-binding activity strongly suggests that T5/7 phosphorylation does not alter the intrinsic chaperone activity of Hsp90 $\alpha$. Thus, at this time, it seems possible that T5/7 phosphorylation of Hsp90 $\alpha$ is entirely fortuitous. Inasmuch as multiple components of repair foci have been shown to be Hsp90 clients (Pennisi et al., 2015), including DNA-PK (Solier et al., 2012), ATR (Ha et al., 2011) and ATM (Pennisi et al., 2017). The Hsp90 $\alpha$ TQTQ motif clearly accommodates all of the phosphatidylinositol 3-kinase-related kinases as a substrate for phosphorylation (Solier et al., 2012), and these clients may phosphorylate the Hsp90 $\alpha$ chaperone because it is bound to them in a chaperone client complex without any effect on $\mathrm{Hsp} 90 \alpha$ chaperone activity.

Because cycling with Hsp90 plays a major role in proteostasis of a number of oncoproteins, there has been considerable interest in developing Hsp90 inhibitors as potential drugs for the treatment of cancers driven by Hsp90 clients, with one focus being development of isoform-specific inhibitors (Trepel et al., 2010). The requirement that Hsp90 functions only as a dimer (Wayne and Bolon, 2007) and the finding that a client protein can exist in complex with an $\operatorname{Hsp} 90 \alpha \beta$ heterodimer in which the inactivation of one member of the dimer would inactivate the complex may diminish the attractiveness of isoform-specific inhibitors.

\section{Acknowledgments}

We thank Yee Sun Tan for constructing the human Hsp90 $\alpha$ T5/7A and T5/7D mutations. 


\section{Authorship Contributions}

Participated in research design: Pratt, Nyati, Osawa.

Conducted experiments: Morishima, Yoshimura, Lau.

Contributed new reagents or analytic tools: Mehta, Southworth, Nyati.

Wrote or contributed to the writing of the manuscript: Lawrence, Pratt, Osawa.

\section{References}

Bharadwaj S, Ali A, and Ovsenek N (1999) Multiple components of the HSP90 chaperone complex function in regulation of heat shock factor 1 in vivo. Mol Cell Biol 19:8033-8041.

Chadli A, Felts SJ, and Toft DO (2008) GCUNC45 is the first Hsp90 co-chaperone to show $\alpha / \beta$ isoform specificity. J Biol Chem 283:9509-9512.

Ciccia A and Elledge SJ (2010) The DNA damage response: making it safe to play with knives. Mol Cell 40:179-204.

Elaimy AL, Ahsan A, Marsh K, Pratt WB, Ray D, Lawrence TS, and Nyati MK (2016) ATM is the primary kinase responsible for phosphorylation of Hsp90 $\alpha$ after ionizing radiation. Oncotarget 7:82450-82457.

Grad I, Cederroth CR, Walicki J, Grey C, Barluenga S, Winssinger N, De Massy B, Nef S, and Picard D (2010) The molecular chaperone Hsp90 $\alpha$ is required for meiotic progression of spermatocytes beyond pachytene in the mouse. PLoS One 5:e15770.

Ha K, Fiskus W, Rao R, Balusu R, Venkannagari S, Nalabothula NR, and Bhalla $\mathrm{KN}$ (2011) Hsp90 inhibitor-mediated disruption of chaperone association of ATR with hsp90 sensitizes cancer cells to DNA damage. Mol Cancer Ther 10 1194-1206.

Kanelakis KC and Pratt WB (2003) Regulation of glucocorticoid receptor ligandbinding activity by the hsp90/hsp70-based chaperone machinery. Methods Enzymol 364:159-173.

Kirschke E, Goswami D, Southworth D, Griffin PR, and Agard DA (2014) Glucocorticoid receptor function regulated by coordinated action of the Hsp90 and Hsp70 chaperone cycles. Cell 157:1685-1697.

Lees-Miller SP and Anderson CW (1989) The human double-stranded DNA-activated protein kinase phosphorylates the $90-\mathrm{kDa}$ heat-shock protein, hsp90 $\alpha$ at two $\mathrm{NH}_{2}$-terminal threonine residues. J Biol Chem 264:17275-17280.

Matsuoka S, Ballif BA, Smogorzewska A, McDonald ER, III, Hurov KE, Luo J, Bakalarski CE, Zhao Z, Solimini N, Lerenthal Y, et al. (2007) ATM and ATR substrate analysis reveals extensive protein networks responsive to DNA damage. Science 316:1160-1166.

Mendel DB and Ortí E (1988) Isoform composition and stoichiometry of the $\sim 90 \mathrm{kDa}$ heat shock protein associated with glucocorticoid receptors. J Biol Chem 263: 6695-6702.

Millson SH, Truman AW, Rácz A, Hu B, Panaretou B, Nuttall J, Mollapour M, Söti C, and Piper PW (2007) Expressed as the sole Hsp90 of yeast, the $\alpha$ and $\beta$ isoforms of human Hsp90 differ with regard to their capacities for activation of certain client proteins, whereas only Hsp90 $\beta$ generates sensitivity to the Hsp90 inhibitor radicicol. FEBS J 274:4453-4463.

Minami Y, Kimura Y, Kawasaki H, Suzuki K, and Yahara I (1994) The carboxyterminal region of mammalian HSP90 is required for its dimerization and function in vivo. Mol Cell Biol 14:1459-1464.

Morishima Y, Murphy PJM, Li D-P, Sanchez ER, and Pratt WB (2000) Stepwise assembly of a glucocorticoid receptor hsp90 heterocomplex resolves two sequential ATP-dependent events involving first hsp70 and then hsp90 in opening of the steroid binding pocket. J Biol Chem 275:18054-18060.
Murphy PJM, Morishima Y, Chen H, Galigniana MD, Mansfield JF, Simons SS Jr., and Pratt WB (2003) Visualization and mechanism of assembly of a glucocorticoid receptor $\bullet$ Hsp70 complex that is primed for subsequent Hsp90-dependent opening of the steroid binding cleft. J Biol Chem 278:34764-34773.

Peng HM, Morishima Y, Pratt WB, and Osawa Y (2012) Modulation of heme/substrate binding cleft of neuronal nitric-oxide synthase (nNOS) regulates binding of Hsp90 and Hsp70 proteins and nNOS ubiquitination. J Biol Chem 287:1556-1565.

Pennisi R, Antoccia A, Leone S, Ascenzi P, and di Masi A (2017) Hsp90 $\alpha$ regulates ATM and NBN functions in sensing and repair of DNA double-strand breaks. FEBS J 284:2378-2395.

Pennisi R, Ascenzi P, and di Masi A (2015) Hsp90: a new player in DNA repair? Biomolecules 5:2589-2618.

Pratt WB, Morishima Y, and Osawa Y (2008) The Hsp90 chaperone machinery regulates signaling by modulating ligand binding clefts. J Biol Chem 283 22885-22889.

Pratt WB and Toft DO (2003) Regulation of signaling protein function and trafficking by the hsp90/hsp70-based chaperone machinery. Exp Biol Med (Maywood) 228: 111-133.

Quanz M, Herbette A, Sayarath M, de Koning L, Dubois T, Sun JS, and Dutreix M (2012) Heat shock protein $90 \alpha(\mathrm{Hsp} 90 \alpha)$ is phosphorylated in response to DNA damage and accumulates in repair foci. J Biol Chem 287:8803-8815.

Richter K, Muschler P, Hainzl O, and Buchner J (2001) Coordinated ATP hydrolysis by the Hsp90 dimer. J Biol Chem 276:33689-33696.

Richter K, Soroka J, Skalniak L, Leskovar A, Hessling M, Reinstein J, and Buchner J (2008) Conserved conformational changes in the ATPase cycle of human Hsp90. J Biol Chem 283:17757-17765.

Solier S, Kohn KW, Scroggins B, Xu W, Trepel J, Neckers L, and Pommier Y (2012) Heat shock protein $90 \alpha$ (HSP90 $\alpha$ ), a substrate and chaperone of DNA-PK necessary for the apoptotic response. Proc Natl Acad Sci USA 109:12866-12872.

Southworth DR and Agard DA (2008) Species-dependent ensembles of conserved conformational states define the Hsp90 chaperone ATPase cycle. Mol Cell 32: $631-640$

Southworth DR and Agard DA (2011) Client-loading conformation of the Hsp90 molecular chaperone revealed in the cryo-EM structure of the human Hsp90:Hop complex. Mol Cell 42:771-781.

Sreedhar AS, Kalmár E, Csermely P, and Shen YF (2004) Hsp90 isoforms: functions, expression and clinical importance. FEBS Lett 562:11-15.

Sullivan WP and Toft DO (1993) Mutational analysis of hsp90 binding to the progesterone receptor. J Biol Chem 268:20373-20379.

Taipale M, Jarosz DF, and Lindquist S (2010) HSP90 at the hub of protein homeostasis: emerging mechanistic insights. Nat Rev Mol Cell Biol 11:515-528.

Trepel J, Mollapour M, Giaccone G, and Neckers L (2010) Targeting the dynamic HSP90 complex in cancer. Nat Rev Cancer 10:537-549.

Voss AK, Thomas T, and Gruss P (2000) Mice lacking HSP90beta fail to develop a placental labyrinth. Development 127:1-11.

Wayne N and Bolon DN (2007) Dimerization of Hsp90 is required for in vivo function. Design and analysis of monomers and dimers. J Biol Chem 282:35386-35395.

Zou J, Guo Y, Guettouche T, Smith DF, and Voellmy R (1998) Repression of heat shock transcription factor HSF1 activation by HSP90 (HSP90 complex) that forms a stress-sensitive complex with HSF1. Cell 94:471-480.

Address correspondence to: Dr. Yoichi Osawa, Department of Pharmacology, University of Michigan Medical School, 1301 Medical Center Drive, 1301A Medical Science Research Building III, Ann Arbor, MI 48109-0632. E-mail: osawa@umich.edu 